University of Wollongong

Research Online

Faculty of Engineering - Papers (Archive)

Faculty of Engineering and Information

Sciences

April 1995

\title{
Theory of photoluminescence of magnetopolarons in quantum wells containing high densities of electrons
}

\author{
L. Swierkowski \\ University of New South Wales \\ J. Szymanski \\ Telecom Australia Research Laboratories, Clayton, Australia \\ Philip E. Simmonds \\ University of Wollongong, simmonds@uow.edu.au \\ T. A. Fisher \\ University of Sheffield, UK \\ M. S. Skolnick \\ University of Sheffield, UK
}

Follow this and additional works at: https://ro.uow.edu.au/engpapers

Part of the Engineering Commons

https://ro.uow.edu.au/engpapers/261

\section{Recommended Citation}

Swierkowski, L.; Szymanski, J.; Simmonds, Philip E.; Fisher, T. A.; and Skolnick, M. S.: Theory of photoluminescence of magnetopolarons in quantum wells containing high densities of electrons 1995. https://ro.uow.edu.au/engpapers/261 


\title{
Theory of photoluminescence of magnetopolarons in quantum wells containing high densities of electrons
}

\author{
L. Świerkowski \\ School of Physics, The University of New South Wales, Sydney 2052, Australia \\ J. Szymański \\ Telecom Australia Research Laboratories, 770 Blackburn Road, Clayton, Victoria 3168, Australia \\ P. E. Simmonds \\ Department of Physics, University of Wollongong, Wollongong, New South Wales 2522, Australia
}

T. A. Fisher and M. S. Skolnick

Department of Physics, University of Sheffield, Sheffield S3 7RH, United Kingdom

(Received 20 October 1994)

\begin{abstract}
We present a theory of photoluminescence in the presence of a quantizing magnetic field for a quasi-two-dimensional electron gas interacting with the lattice assuming a weak Fröhlich interaction with bulk longitudinal optical (LO) phonons. Unlike in the conventional cyclotron resonance, the calculated photoluminescence spectrum for high electron concentrations exhibits strong renormalization due to the resonant electron-phonon coupling between two quasiholes in conduction Landau levels whenever the LO-phonon energy is close to the energy difference between any two occupied Landau levels. The high electron concentration is necessary to push the Fermi energy well above that of LO phonons. The screening of the electron-phonon interaction was included within the static random-phase approximation and the finite extent of the electron wave function in the quantum well was accounted for. The calculated spectra are in excellent agreement with experimental photoluminescence data for $\mathrm{GaAs} / \mathrm{In}_{x} \mathrm{Ga}_{1-x} \mathrm{As} / \mathrm{Al}_{y} \mathrm{Ga}_{1-y} \mathrm{As}$ quantum wells.
\end{abstract}

\section{INTRODUCTION}

The motion of electrons and holes in polar semiconductors is affected by their coupling to the polarization field of longitudinal-optical (LO) phonons. The effects of this electron-phonon interaction has the most pronounced effect in the presence of a quantizing magnetic field when the multiple of the cyclotron frequency $\omega_{c}$ approaches the optical phonon frequency $\omega_{\text {LO }}$. During the last decade, this resonant polaron effect has been the subject of considerable interest in two-dimensional systems both experimentally and theoretically. Most effort has been devoted to the observation and theoretical description of the two-dimensional (2D) resonant polaron effect in the cyclotron resonance in moderately populated conduction bands, despite the fact that cyclotron resonance does not readily allow direct observation of the line splitting. In contrast, in samples with high electron concentration $\left(E_{F}>\hbar \omega_{\mathrm{LO}}\right)$, the resonant polaron effect can be directly observed as the splitting of the magnetoluminescence lines. We calculate the magnetoluminescence spectrum for $\mathrm{Al}_{x} \mathrm{Ga}_{1-x} \mathrm{As} / \mathrm{In}_{y} \mathrm{Ga}_{1-y} \mathrm{As} / \mathrm{GaAs}$ quantum wells and compare the results with the latest experiments. ${ }^{1}$

Theoretical calculations of the cyclotron absorption based on a one-polaron approximation ${ }^{2-4}$ predict enhancement of the resonant polaronic effect in twodimensional systems when the magnetic field is perpendicular to the semiconductor interface. This is due to the lack of momentum conservation in the direction per-

pendicular to the interface. Experimentally, it has been found that depending of the particular sample the polaronic effects in cyclotron resonance are enhanced in some situations ${ }^{5-8}$ and reduced in others ${ }^{6,8-12}$ as compared to equivalent experimental studies of bulk 3D semiconductor systems. In real quasi-two-dimensional systems, the finite extension of the wave function perpendicular to the interface tends to reduce the effective coupling strength $^{13-15}$ up to its three-dimensional limit. A further reduction is caused by the presence of electrons in the conduction band $\left(\sim 10^{11} \mathrm{~cm}^{-2}\right)$, which leads to both Pauli blocking of scattering states necessary for the polaron interaction to be effective (occupational effect) and screening of the electron-phonon interaction. ${ }^{16,17}$

Cyclotron resonance experiments have important limitation as far as investigation of the resonant polaron is concerned. The lattice absorption (reststrahlen band) limits the possibility of direct observation of resonant splitting and as a result in the majority of experiments, only the relatively small changes of the cyclotron effective mass below and above the resonance have been observed. Occasionally, while the splitting cannot be observed directly, ${ }^{8}$ the theoretical fit to the data can give the effective mass within the reststrahlen band. ${ }^{18} \mathrm{An}$ important exception here is InSe system where the electronphonon interaction is sufficiently large to push the cyclotron resonance lines outside the reststrahlen band. ${ }^{19,20}$ The resonant splitting of absorption line can also be observed for impurity states. ${ }^{21}$ 
The lattice absorption problem can be entirely avoided by performing interband optical experiments ${ }^{22}$ in which photon energy is much larger then the cyclotron energy. However, the occupational effect in interband absorption experiments (if carried out in the presence of significant free carrier densities) influences measurements in a manner similar to that found in cyclotron resonance experiments. It quenches the resonant polaron coupling by blocking emission of LO phonons when the filling factor of the lower Landau level approaches one. This phenomenon restricts usefulness of absorption methods to the study of polaron coupling between nearly empty levels if the resonant coupling is to be observed at close to its full strength.

On the other hand, as demonstrated in Ref. 1, interband magnetoluminescence experiments reveal strong polaron coupling between quasiholes in filled conduction Landau levels and the presence of electrons at high densities is essential so that $E_{F}>\hbar \omega_{\mathrm{LO}}$. The resonant splitting can be directly observed: experiments in modulation doped $\mathrm{Al}_{x} \mathrm{Ga}_{1-x} \mathrm{As} / \mathrm{In}_{y} \mathrm{Ga}_{1-y} \mathrm{As} / \mathrm{GaAs}$ quantum wells ${ }^{1}$ which are the motivation of our calculation, produce a rich spectrum of anomalies due to the resonant polaron coupling in magnetic fields satisfying $\omega_{\mathrm{LO}} \approx \mathcal{N} \omega_{c}$, where $\mathcal{N}$ is a positive integer.

The comparison of theory and experiments in the case of cyclotron resonance experiments was restricted to the comparison of polaron effective masses. In the case of the interband magnetoluminescence experiments, the spectrum is qualitatively changed and it is possible to directly compare the splitting of observed lines. In Sec. II, we employed memory function formalism to calculate the photoluminescence spectrum; memory formalism was used before to calculate polaronic effect in cyclotron resonance absorption. ${ }^{16}$ The calculation of the screening is outlined in Sec. III. Our numerical results are discussed and compared with experiment in Sec. IV.

\section{CALCULATION OF PHOTOLUMINESCENCE}

The system we consider consists of a single quantum well confining the motion of electron in the $z$ direction, in a strong magnetic field perpendicular to the layer. The concentration of conduction electrons in the well is assumed to be such that only the lowest subband is occupied, so that the motion of electrons in the $z$ direction is described by a single envelope function $\varphi(z)$ in the effective mass approximation.

In the presence of a single hole in the valence band, the probability of emission of a photon with the wave vector $\boldsymbol{Q}$, energy $\hbar \omega$ and polarization $\boldsymbol{e}$ can be obtained from the Fermi Golden rule and recast in the following form:

$$
\begin{aligned}
P(\omega)= & -\frac{2}{\hbar^{2}} \operatorname{Im} \sum_{N k_{x}, N^{\prime} k_{x}^{\prime}} B_{N^{\prime} k_{x}^{\prime}, v}(\boldsymbol{Q}, \boldsymbol{e}) B_{N k_{x}, v}^{*}(\boldsymbol{Q}, \boldsymbol{e}) \\
& \times(-i) \int_{0}^{\infty} d t e^{i z t}\left\langle\left\langle c_{N^{\prime} k_{x}^{\prime}}^{\dagger}(t) c_{N k_{x}}(0)\right\rangle\right\rangle,
\end{aligned}
$$

where $B_{n k_{x}, v}^{*}(\boldsymbol{Q}, \boldsymbol{e})$ is the matrix element of the electronphoton interaction between the conduction state $N k_{x}$ and the valence state $v, N k_{x}$ are the conduction electron quantum numbers (Landau gauge), $\hbar z=-\hbar \omega+E_{g}-\epsilon_{v}+$ $i \varepsilon, \epsilon_{v}<0$ is the valence electron energy measured from the top of the valence band, and $c_{N k_{x}}$ is the annihilation operator of conduction electrons. $\langle\langle\rangle\rangle$ is the canonical average over the system of electrons and phonons.

The evolution of $c_{N k_{x}}(t)$ is governed by the electronphonon Hamiltonian:

$$
\begin{aligned}
H= & \sum_{N k_{x}} \hbar \omega_{N} c_{N k_{x}}^{\dagger} c_{N k_{x}}+\hbar \omega_{L O} \sum_{\boldsymbol{q}} b_{\boldsymbol{q}}^{\dagger} b_{\boldsymbol{q}} \\
& +\sum_{N N^{\prime} k_{x} \boldsymbol{q}} i V_{N^{\prime} N}\left(k_{x}, \boldsymbol{q}\right) c_{N^{\prime} k_{x}+\boldsymbol{q}_{x}}^{\dagger} c_{N k_{x}}\left(b_{\boldsymbol{q}}-b_{-\boldsymbol{q}}^{\dagger}\right),
\end{aligned}
$$

where

$$
\begin{gathered}
V_{N^{\prime} N}\left(k_{x}, \boldsymbol{q}\right)=V_{\boldsymbol{q}} \int d_{3} r \psi_{N^{\prime} k_{x}+q_{x}}^{*}(\boldsymbol{r}) e^{i \boldsymbol{q} \cdot \boldsymbol{r}} \psi_{N k_{x}}(\boldsymbol{r}) \\
V_{\boldsymbol{q}}=\hbar \omega_{\mathrm{LO}} \sqrt{\frac{4 \pi \alpha}{\Omega}}\left(\frac{\hbar}{2 m_{e}^{*} \omega_{\mathrm{LO}}}\right)^{\frac{1}{4}} \frac{1}{q} \\
\alpha=\frac{1}{2}\left(\frac{1}{\varepsilon_{\infty}}-\frac{1}{\varepsilon_{0}}\right)\left(\frac{2 m_{e}^{*} \omega_{\mathrm{LO}}}{\hbar}\right)^{\frac{1}{2}} \\
\omega_{N}=\omega_{c}\left(N+\frac{1}{2}\right)
\end{gathered}
$$

$\psi_{N k_{x}}(\boldsymbol{r})$ is the electron wave function in the effective mass approximation, $b_{\boldsymbol{q}}$ is the phonon annihilation operator, $\Omega$ is the volume of the system, $\varepsilon_{0}\left(\varepsilon_{\infty}\right)$ is the static (high frequency) dielectric constant of the host.

Equation (1) for the photoluminescence function can be simplified due to the fact that $\left\langle\left\langle c_{N^{\prime} k_{x}^{\prime}}^{\dagger}(t) c_{N k_{x}}(0)\right\rangle\right\rangle$ is by symmetry diagonal in $k_{x} k_{x}^{\prime}$ and is also diagonal in $N N^{\prime}$ in the lowest order in the phonon-electron interaction [cf. Eq. (17)]. In this case,

$$
P(\omega)=-\frac{2}{\hbar^{2}} \operatorname{Im} \sum_{N k_{x}}\left|B_{N k_{x}, v}(\boldsymbol{Q}, \boldsymbol{e})\right|^{2} G_{N}(z),
$$

where $G_{N}(z)$ is the Laplace transform of the propagator of a quasihole in the conduction band,

$$
\begin{aligned}
G_{N}(z) & =-i \int_{0}^{\infty} d t e^{i z t}\left\langle\left\langle c_{N k_{x}}^{\dagger}(t) c_{N k_{x}}(0)\right\rangle\right\rangle \\
& =\left\langle\left\langle c_{N k_{x}}^{\dagger} \frac{1}{z-L} c_{N k_{x}}\right\rangle\right\rangle .
\end{aligned}
$$

We have introduced the Liouville operator $L$,

$$
L A=\frac{1}{\hbar}[H, A]
$$

where [ ] is the commutator.

In the spirit of the memory function approach, we define the scalar product of two operators, $A$ and $B$,

$$
\langle A \mid B\rangle=\left\langle\left\langle A^{\dagger} B\right\rangle\right\rangle
$$


The scalar product of two annihilation operators is proportional to the Fermi distribution function $f_{N}$ :

$$
\left\langle c_{N k_{x}} \mid c_{N^{\prime} k_{x}^{\prime}}\right\rangle=f_{N} \delta_{N N^{\prime}} \delta_{k_{x} k_{x}^{\prime}} .
$$

We can introduce a projection operator onto the conduction electrons states,

$$
P=\sum_{N k_{x}}\left|c_{N k_{x}}\right\rangle \frac{1}{f_{N}}\left\langle c_{N k_{x}}\right|
$$

and write

$$
\begin{aligned}
G_{N}(z) & =\left\langle c_{N k_{x}}\left|P \frac{1}{z-L} P\right| c_{N k_{x}}\right\rangle \\
& =\left\langle c_{N k_{x}}\left|\frac{1}{z-P L P-\Sigma(z)}\right| c_{N k_{x}}\right\rangle,
\end{aligned}
$$

where

$$
\Sigma(z)=P L Q \frac{1}{z-Q L Q} Q L P
$$

and $Q=1-P$.

We calculate both $P L P$ and $\Sigma(z)$ in the lowest order in the electron-phonon interaction and obtain

$$
\left\langle c_{N k_{x}}|L| c_{N^{\prime} k_{x}}\right\rangle=-\omega_{N} f_{N} \delta_{N N^{\prime}}
$$

and

$$
\begin{aligned}
\left\langle c_{N^{\prime} k_{x}}\left|L Q \frac{1}{z-Q L Q} Q L\right| c_{N k_{x}}\right\rangle & \\
= & \frac{1}{\hbar^{2}} \sum_{N^{\prime \prime} \boldsymbol{q}} V_{N^{\prime} N^{\prime \prime}}^{*}\left(k_{x}, \boldsymbol{q}\right) f_{N^{\prime \prime}}\left(\frac{n_{\boldsymbol{q}}}{z+\omega_{N^{\prime \prime}}+\omega_{\mathrm{LO}}}\right. \\
& \left.+\frac{n_{\boldsymbol{q}}+1}{z+\omega_{N^{\prime \prime}}-\omega_{\mathrm{LO}}}\right) V_{N^{\prime \prime} N}\left(k_{x}, \boldsymbol{q}\right),
\end{aligned}
$$

where $n_{q}$ is the phonon occupation number. The integration over the angles of $\boldsymbol{q}$ produces $\delta_{N N^{\prime}}$ in Eq. (17), justifying Eq. (7). Using Eq. (3) and neglecting the terms $\sim n_{q}\left(k_{B} T<<\hbar \omega_{\mathrm{LO}}\right)$ in Eq. (17), we obtain the final expression for $G_{N}(z)$ :

$$
G_{N}(z)=\frac{f_{N}}{z+\omega_{N}-\Sigma_{N}(z)}
$$

where

$$
\Sigma_{N}(z)=\alpha \omega_{\mathrm{LO}}^{2} \sqrt{\frac{\omega_{c}}{\omega_{\mathrm{LO}}}} \sum_{N^{\prime \prime}} \frac{f_{N^{\prime \prime}} C_{N N^{\prime \prime}}}{z+\omega_{N^{\prime \prime}}-\omega_{\mathrm{LO}}},
$$

and

$$
\begin{aligned}
C_{N N^{\prime \prime}}= & \frac{l}{\pi \sqrt{2}} \frac{N !}{N^{\prime \prime} !} \int_{-\infty}^{\infty} d q_{z} \int_{0}^{\infty} d q_{\|} \frac{q_{\|}}{q_{\|}^{2}+q_{z}^{2}}\left|g\left(q_{z}\right)\right|^{2} \\
& \times\left(\frac{l^{2} q_{\|}^{2}}{2}\right)^{N^{\prime \prime}-N} e^{-\frac{t^{2} q_{\|}^{2}}{2}}\left[L_{N}^{\left(N^{\prime \prime}-N\right)}\left(\frac{l^{2} q_{\|}^{2}}{2}\right)\right]^{2} .
\end{aligned}
$$

The magnetic length $l=\sqrt{\hbar c / e B}, L_{n}^{(m)}(x)$ are generalized Laguerre polynomials, ${ }^{23} q_{\|}$is the component of the momentum $\boldsymbol{q}$ parallel to the layer and

$$
g\left(q_{z}\right)=\int_{-\infty}^{\infty} d z|\varphi(z)|^{2} e^{i q_{z} z}
$$

is due to the finite width of the electron layer.

The position of the photoluminescence lines can be obtained directly from singularities of $G_{N}(z)$ given by Eq. (18) without the knowledge of the coefficients $B_{N k_{x}, v}(\boldsymbol{q}, \boldsymbol{e})$. These coefficients are only necessary if we want to find selection rules and the relative strength of the lines. In the samples we describe, however, some selection rules (e.g., $\Delta N=0$ ) are broken by disorder ${ }^{1}$ and since we do not include disorder in our approach, we concentrate here on calculating the energies of the optical transitions. It is worth noting that only occupied Landau levels contribute to the self-energy Eq. (19) and, consequently, the resonant magnetopolaron effect can occur only if $E_{F}>\hbar \omega_{\mathrm{LO}}$.

\section{SCREENING}

Since it has been demonstrated that dynamic screening of the electron-phonon interaction gives results essentially identical to those obtained with static screening, ${ }^{24}$ we limit ourselves to the numerically simpler calculations within the static screening approximation. The static screening of the electron-phonon interaction is introduced by replacing $V_{\boldsymbol{q}}$ with $V_{\boldsymbol{q}} / \varepsilon\left(q_{\|}\right)$in Eq. (3). We have calculated the static dielectric function $\varepsilon\left(q_{\|}\right)$in the randomphase approximation and obtained

$$
\varepsilon\left(q_{\|}\right)=1+\frac{2 \pi e^{2}}{q \varepsilon_{\infty}} f_{r}\left(q_{\|}\right) \chi^{(0)}\left(q_{\|}\right) .
$$

$\chi^{(0)}\left(q_{\|}\right)$is the response function for the ideal twodimensional noninteracting electron gas in a magnetic field perpendicular to the layer ${ }^{25,26}$

$$
\begin{aligned}
\chi^{(0)}\left(q_{\|}\right)= & \frac{m_{e}^{*}}{\hbar^{2}} \frac{2}{\pi^{2}} \int_{0}^{\pi} d t t \sin \left(\frac{l^{2} q_{\|}^{2}}{2} \sin t\right) e^{-\frac{l^{2} q_{\|}^{2}}{2}(1+\cos t)} \\
& \times\left\{L_{N}^{(1)}\left[l^{2} q_{\|}^{2}(1+\cos t)\right]\right. \\
& \left.-\left(1-f_{N}\right) L_{N}\left[l^{2} q_{\|}^{2}(1+\cos t)\right]\right\}
\end{aligned}
$$

and the form factor

$$
\begin{aligned}
f_{r}\left(q_{\|}\right) & =\int_{-\infty}^{\infty} d z \int_{-\infty}^{\infty} d z^{\prime} e^{-q_{\|}\left|z-z^{\prime}\right|}|\varphi(z)|^{2}\left|\varphi\left(z^{\prime}\right)\right|^{2} \\
& =\frac{q_{\|}}{\pi} \int_{-\infty}^{\infty} d q_{z} \frac{\left|g\left(q_{z}\right)\right|^{2}}{q_{\|}^{2}+q_{z}^{2}}
\end{aligned}
$$

where $g\left(q_{z}\right)$ is given by Eq. (21). In Eq. (23), $N$ is the principal quantum number of the highest Landau level containing electrons. It is given by $N=\operatorname{Int}(\nu / 2)$, where $\operatorname{Int}(x)$ means the integer part of $x$ and $\nu=2 \pi l^{2} n$ is the filling factor corresponding to electron concentration $n$. 


\section{NUMERICAL RESULTS}

The resonant magnetopolaron effects in the photoluminescence process can be understood in the following way. The initial state of the system consists of a number of occupied Landau levels below the Fermi level in the conduction band and a single hole in the valence band. Under the resonance condition $\omega_{\mathrm{LO}} \approx \mathcal{N} \omega_{c}$ at least two final states are possible if $E_{F}>\hbar \omega_{\mathrm{LO}}$. Each one contains a photon of energy $\hbar \omega$ and (i) a quasihole in the $N_{e}$ th conduction Landau level or (ii) a quasihole in the $N_{e}^{\prime}=\mathcal{N}+N_{e}$ Landau level and an optical phonon of energy $\hbar \omega_{\text {LO }}$ provided both $N_{e}$ and $N_{e}^{\prime}$ Landau levels lie below the Fermi level. These two nearly degenerate states are strongly mixed by the electron-phonon interaction, which lifts degeneracy leading to the resonant magnetopolaron splitting.

The important difference between these magnetophotoluminescence experiments and the cyclotron resonance experiments is that here two quasiholes from different filled conduction Landau levels are coupled by the LO phonon, while in the cyclotron resonance two electrons from nearly empty conduction Landau levels are coupled.

In the numerical work we included the effects of the valence band nonparabolicity ${ }^{27}$ by writing the unperturbed transition energy in the form

$$
\begin{aligned}
\hbar \omega= & E_{g}+\hbar \omega_{c}\left(N_{e}+\frac{1}{2}\right) \\
& +\frac{\hbar e B}{m_{h}^{*} c}\left(N_{h}+\frac{1}{2}\right)\left[1-\beta \frac{\hbar e B}{m_{h}^{*} c}\left(N_{h}+\frac{1}{2}\right)\right],
\end{aligned}
$$

where $E_{g}$ is the band gap, $m_{e}^{*}\left(m_{h}^{*}\right)$ is electron (hole) effective mass, and $\beta$ describes the valence band nonparabolicity. [The upper Landau fan in Figs. 1 and 2 is obtained by considering only the highest valence Landau level, i.e., by setting $N_{h}=0$ in Eq. (25); the lower Landau fan is the phonon replica of the upper one.]

In Fig. 1, we present the comparison between theoretical positions of photoluminescence lines and experimental points for an asymmetric modulation-doped $\mathrm{Al}_{y} \mathrm{Ga}_{1-y} \mathrm{As} / \mathrm{In}_{x} \mathrm{Ga}_{1-x} \mathrm{As} / \mathrm{GaAs}(y \approx 0.23, x \approx 0.1)$ strained-layer quantum well (150 $\AA$ wide) with electron concentration $n_{s}=1.6 \times 10^{12} \mathrm{~cm}^{-2}$ corresponding to $E_{F} \approx 56 \mathrm{meV} .{ }^{1}$ In the calculation we used the following values for the parameters: $m_{e}^{*}=0.069 m_{0}, m_{h}^{*}=0.09 m_{0}$. $\beta=0.031 \mathrm{meV}^{-1}, \hbar \omega_{\mathrm{LO}}=36.7 \mathrm{meV}$, and the dimensionless strength of the Fröhlich interaction, $\alpha=0.068$ [cf. Eq. (5)]. Only the absolute energy scale was adjusted by varying $E_{g}$ in Eq. (25): the best fit was obtained for $E_{g}=1.40 \mathrm{eV}$. It is worth noting that relative energies, in particular resonant polaron splittings, do not depend on the value of $E_{g}$.

Figure 2 gives a similar comparison for a different sample with $n_{s}=1.43 \times 10^{12} \mathrm{~cm}^{-2}\left(E_{F} \approx 50 \mathrm{meV}\right) .{ }^{28} \mathrm{In}$ this case we took $E_{g}=1.41 \mathrm{eV}$. The agreement of experimental points and theoretical curves is excellent. The deviation of the experimental points at the highest energies from the expected straight line behavior in Fig. 2 is probably due to the effect of depopulation of the highest Landau level ${ }^{29}$ and is not included in our calculation.

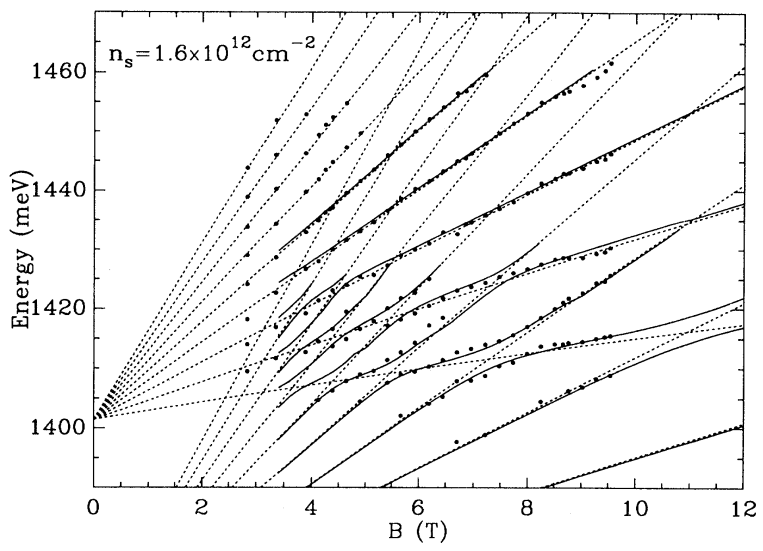

FIG. 1. Predicted positions (solid lines) of the photoluminescence lines and the experimental results for electron concentration $n_{s}=1.60 \times 10^{12} \mathrm{~cm}^{-2}$ (Ref. 1) (solid circles). Dotted lines-unperturbed Landau level fan and its optical phonon replica. (Note that only one hole level takes part in the photoluminescence transitions.)

\section{DISCUSSION}

Resonant splitting. The resonant magnetopolaron splitting can be clearly seen in Figs. 1 and 2 for the magnetic field satisfying the resonant conditions $\omega_{\mathrm{LO}} \approx \mathcal{N} \omega_{c}$ with $\mathcal{N}=2$ to 6 . At the exact resonance, the splitting $\Delta_{\mathcal{N}}$ of the photoluminescence line can easily be evaluated in an analytic form. From Eqs. (3), (18), and (19) we get

$$
\Delta_{\mathcal{N}}=2 \hbar \omega_{\mathrm{LO}} \alpha^{1 / 2} C_{N_{e} N_{e}^{\prime}}^{1 / 2} / \mathcal{N}^{1 / 4}
$$

where $N_{e}^{\prime}-N_{e}=\mathcal{N}$. Thus, in agreement with experiment, the resonant splitting is larger for small $\mathcal{N}$, i.e., for high magnetic field than it is for lower fields. Note that in Eq. (26) the dependence of the magnitude of the splitting on magnetic field is contained in both the coef-

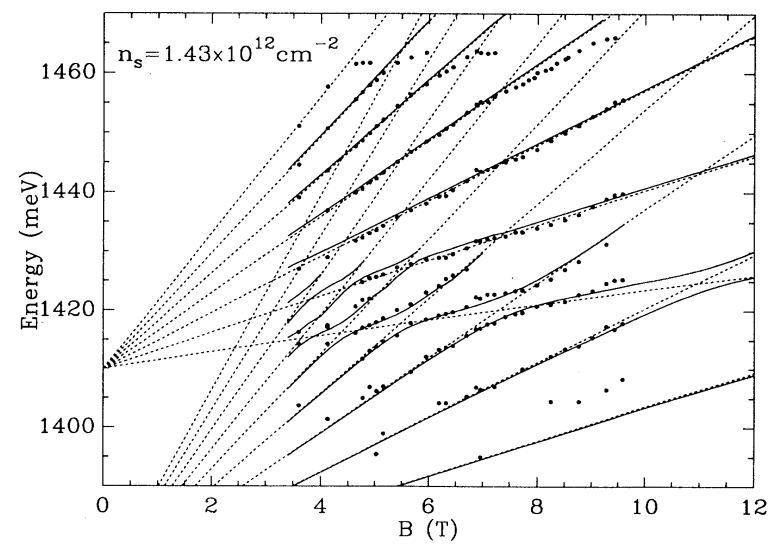

FIG. 2. As in Fig. 1, but for lower electron concentration $n_{s}=1.43 \times 10^{12} \mathrm{~cm}^{-2}$ (Ref. 23). 
ficients $C_{N_{e} N^{\prime}}$ and, through the resonance condition, in the factor $\mathcal{N}^{-1 / 4}$.

Screening and occupational effects. Magnetoluminescence provides a useful experimental tool to separate effects of screening from occupational effects on magnetopolaron coupling, in contrast with cyclotron resonance experiments where it is difficult. ${ }^{8,17}$ If both levels $N_{e}$ and $N_{e}^{\prime}$ are occupied, only the screening plays a role. This is the case for the $\left(N_{e}=0, N_{e}^{\prime}=3\right)$ resonance from Fig. 1 which has been magnified in Fig. 3 . The solid line gives the result with the static screening calculated in the random-phase approximation, while the dashed line was obtained by ignoring the screening. In the latter case, the resonance polaron splitting becomes $\sim 50 \%$ larger in disagreement with the experiment.

The effect of Landau level occupation is clearly visible by looking at the following sequence of $\left(N_{e}, N_{e}^{\prime}\right):(0,3)$, $(1,4)$, and $(2,5)$ in Fig. 1 . The resonant splitting is large for $(0,3)$, becomes small for $(1,4)$, and disappears altogether for $(2,5)$, since the Landau level $N_{e}^{\prime}=5$ is above the Fermi level. (Note that $E_{F} \approx 56 \mathrm{meV}$.)

Confinement. For the ideal two-dimensional system with infinitely narrow quantum wells, the polaron effect can be shown to be much stronger than for the threedimensional case. In real systems, the confinement is not ideal and the polaron effect is diminished approaching its three-dimensional value for very wide quantum wells. We have used self-consistent wave functions in order to obtain correct dependence on the quantum well width (solid lines in Fig. 3). Chain lines show the resonant splitting calculated for the infinitely thin layer without screening. This should be compared with the result for the finite width and no screening (broken lines), showing some sensitivity to the width of the well.

Interestingly, when the screening is included, the results become much less sensitive to the width of the well. Long-broken lines give the result for the infinitely thin layer with the screening included. This somewhat unexpected result can be explained by the fact that the increased strength of the electron-phonon interaction is compensated by the increased strength of the screening. This compensation could be, in principle, tested experimentally.

In summary, we have calculated the energies of twodimensionally confined polarons in the presence of a magnetic field perpendicular to the electron layer assuming a weak Fröhlich interaction with bulk longitudinal-

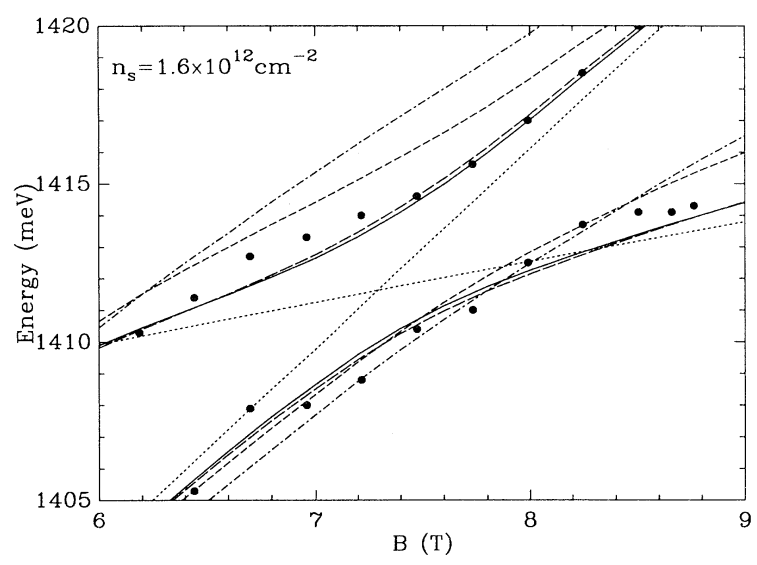

FIG. 3. Magnified region of the $\left(N_{e}=0, N_{e}^{\prime}=3\right)$ resonance from Fig. 1. Additional lines show the results of the calculation without screening (dashed), with the screening for an infinitely thin layer (long-dashed) and without screening for an infinitely thin layer (chain).

optical (LO) phonons. We have considered the case of layers with high electron concentration with the Fermi energy greater than the LO-photon energy, $\hbar \omega_{\mathrm{LO}}$ and included the screening of electron-phonon interaction in the static random-phase approximation. We have used selfconsistent wave functions in the direction perpendicular to the layer and included the nonparabolicity of the hole states. The calculated photoluminescence spectra exhibit strong renormalization due to the resonant electronphonon coupling whenever the LO-phonon energy is close to the energy difference between occupied Landau levels, i.e., $\hbar \omega_{\text {LO }} \approx \mathcal{N} \hbar \omega_{c}$, where $\omega_{c}$ is the cyclotron frequency. This renormalization splits the photoluminescence lines into two components. The calculated spectra are in excellent agreement with experimental data for $\mathrm{GaAs} / \mathrm{In}_{x} \mathrm{Ga}_{1-x} \mathrm{As} / \mathrm{Al}_{y} \mathrm{Ga}_{1-y} \mathrm{As}$ quantum wells.

\section{ACKNOWLEDGMENTS}

We would like to thank Z. W. Gortel for helpful discussions. L.S. acknowledges financial support from the Australian Research Council.
${ }^{1}$ P. E. Simmonds, M. S. Skolnick, T. A. Fisher, K. J. Nash, and R. S. Smith, Phys. Rev. B 45, 9497 (1992).

${ }^{2}$ S. Das Sarma, Phys. Rev. Lett. 52, 859 (1984).

${ }^{3}$ D. M. Larsen, Phys. Rev. B 30, 4807 (1984).

${ }^{4} \mathrm{Wu}$ Xiaoguang, F. M. Peeters, and J. T. Devreese, Phys. Status Solidi B 133, 229 (1986).

${ }^{5}$ M. Horst, U. Merkt, and J. P. Kotthaus, Phys. Rev. Lett. 50, 754 (1983).

${ }^{6}$ J. Singleton, R. J. Nicholas, and F. Nasir, Solid State Commun. 58, 833 (1986).
${ }^{7}$ J. Singleton, R. J. Nicholas, D. C. Rogers, and C. T. B. Foxon, Surf. Sci. 196, 429 (1988).

${ }^{8}$ C. J. G. M. Langerak, J. Singleton, P. J. van der Wel, J. A. A. J. Perenboom, D. J. Barnes, R. J. Nicholas, M. A. Hopkins, and C. T. B. Foxon, Phys. Rev. B 38, 13133 (1988).

${ }^{9}$ M. Horst, U. Merkt, W. Zawadzki, J. C. Maan, and K. Ploog, Solid State Commun. 53, 403 (1985).

${ }^{10}$ H. Sigg, P. Wyder, and J. A. A. J. Perenboom, Phys. Rev. B 31, 5253 (1985). 
${ }^{11}$ R. J. Nicholas, L. C. Brunel, S. Huant, K. Karrai, J. C. Portal, M. A. Brummell, M. Razeghi, K. Y. Cheng, and A. Y. Cho, Phys. Rev. Lett. 55, 883 (1985).

${ }^{12}$ M. Ziesmann, D. Heitmann, and L. L. Chang, Phys. Rev. B 35, 4541 (1987).

${ }^{13}$ S. Das Sarma and A. Madhukar, Phys. Rev. B 22, 2823 (1980).

${ }^{14}$ R. Lassnig and W. Zawadzki, Surf. Sci. 142, 388 (1984).

${ }^{15} \mathrm{Wu}$ Xiaoguang, F. M. Peeters, and J. T. Devreese, Phys. Rev. B 34, 8800 (1986).

${ }^{16}$ Xiaoguang Wu, F. M. Peeters, and J. T. Devreese, Phys. Status Solidi B 143, 581 (1987).

${ }^{17} \mathrm{Wu}$ Xiaoguang, F. M. Peeters, and J. T. Devreese, Phys. Rev. B 40, 4090 (1989).

${ }^{18}$ F. M. Peeters, X.-G. Wu, J. T. Devreese, C. J. G. M. Langerak, J. Singleton, D. J. Barnes, and R. J. Nicholas, Phys. Rev. B 45, 4296 (1992).

${ }^{19}$ F. M. Peeters, X.-G. Wu, J. T. Devreese, M. Watts, R. J. Nicholas, D. F. Howell, L. van Bockstal, F. Herlach, C. J. G. M. Langerak, J. Singleton, and A. Chevy, Surf. Sci. 263, 654 (1992).
${ }^{20}$ R. J. Nicholas, M. Watts, D. F. Howell, F. M. Peeters, X.-G. Wu, J. T. Devreese, L. van Bockstal, F. Herlach, C. J. G. M. Langerak, J. Singleton, and A. Chevy, Phys. Rev. B 45, 12144 (1992).

${ }^{21}$ J. P. Cheng, B. D. McCombe, J. M. Shi, F. M. Peeters, and J. T. Devreese, Phys. Rev. B 48, 7910 (1993).

${ }^{22}$ L. Świerkowski, W. Zawadzki, Y. Guldner, and C. Rigaux, Solid State Commun. 27, 1245 (1978).

${ }^{23}$ M. Abramowitz and I. A. Stegun, Handbook of Mathematical Functions (Dover, New York, 1970).

${ }^{24} \mathrm{Wu}$ Xiaoguang, F. M. Peeters, and J. T. Devreese, Phys. Rev. B 36, 9760 (1987).

${ }^{25}$ L. Świerkowski and J. Szymański (unpublished).

${ }^{26}$ M. L. Glasser, Phys. Rev. B 28, 4387 (1983).

${ }^{27}$ E. D. Jones, S. K. Lyo, I. J. Fritze, J. F. Klem, J. E. Schirber, C. P. Tigges, and T. J. Drummond, Appl. Phys. Lett. 54, 2227 (1989).

${ }^{28}$ P. E. Simmonds, M. S. Skolnick, and T. A. Fisher (unpublished).

${ }^{29}$ M. S. Skolnick, K. J. Nash, M. K. Saker, and S. J. Bass (unpublished). 